\title{
Gen X-Pert MTB/Rif Assay with the Ziehl Nielsen, and Florescence Microscopy Detection Rate of Urinary Tuberculosis Among Suspected and Pulmonary MTB Positive Patients Visiting University of Gondar Specialized Referral Hospital, Northwest Ethiopia.
}

Dessie Eshetie

University of Gondar

Minichil Worku

University of Gondar

Abiye Tigabu

University of Gondar

Melak Aynalem ( $\square$ melak.aynalem1234@gmail.com )

University of Gondar

Nega Berhane

University of Gondar

Research Article

Keywords: Extra-pulmonary tuberculosis, Gene X-pert MTB/RIF, Urinary tract tuberculosis, Ziehl Nielsen microscopy, Florescence microscopy.

Posted Date: August 4th, 2021

DOI: https://doi.org/10.21203/rs.3.rs-763170/v1

License: (1) This work is licensed under a Creative Commons Attribution 4.0 International License.

Read Full License 


\section{Abstract \\ Background}

Tuberculosis is a bacterial infection caused by Mycobacterium tuberculosis remains a major global public health concern. Extra-pulmonary tuberculosis accounts for $15 \%$ of the global tuberculosis burden. Urinary tract tuberculosis is one of the most common and severe forms of extra-pulmonary tuberculosis in clinical practice. Diagnosis of urinary tract tuberculosis by Gen X-pert MTB/RIF assay from developing countries including Ethiopia is limited. Thus, this study was aimed to compare Gene X-pert MTB/RIF assay with the convectional diagnosis methods.

\section{Methods}

A hospital-based cross-sectional study was conducted among confirmed pulmonary tuberculosis and suspected for urinary tract tuberculosis patients at University of Gondar Specialized Referral Hospital from February 2020 to June 2020 G.C. Non-randomized purposive sampling technique was used to select study participants. To detect Mycobacterium tuberculosis, a urine sample was collected. Then, Ziehl Nielsen and fluorescence microscope, Gene X-pert Real-time PCR were performed to detect the Mycobacterium tuberculosis. Sociodemographic, clinical data, and laboratory data were collected and entered into EPI-Info version 3.5.3 and then transferred to SPSS version-20 for analysis. Descriptive statistics were summarized as percentages, means, and standard deviations.

\section{Results}

A total of 64 study participants were enrolled in this study, 64.2\% (41/64) were males and 30\% (19/64) were in the age group of $31-45$ years. Moreover, $71.9 \%$ (46/64) and $57.8 \%(37 / 64)$ study participants were rural residences and illiterate respectively. Among the 64 study participants, 4.69\% (3/64) were positive for urinary tuberculosis by Gene X-expert. However, $1.56 \%(1 / 64)$ was positive by fluorescence microscopy, and there was no urinary tuberculosis detected by Ziehl Nielsen examination method.

\section{Conclusion and recommendation:}

The prevalence of urinary tract Mycobacterium tuberculosis using Gene X-pert and fluorescence microscopy was $4.69 \%(3 / 64)$ and $1.56 \%(1 / 64)$, respectively. Gene X-pert has higher detection rate than the conventional methods. Therefore, it is better to develop a guideline on how to use Gen x-pert for the diagnosis for urinary tract tuberculosis in urine samples.

\section{Background}


Mycobacterium tuberculosis (MTB) complex is a major global public health concern and the first leading cause of death from infectious diseases worldwide (1). One-third of the world's population is estimated to have latent tuberculosis (TB) infection and approximately $10 \%$ of these individuals will develop active TB in their lifetime (2). Coverage of TB preventive treatment is one of the key indicators used to monitor the implementation of the end TB Strategy (3).

The world health organization (WHO) reported 10.4 million new cases of TB and 1.7 million deaths, where $90 \%$ of the cases were from adults ( $60 \%$ male and $35 \%$ women). Among the new cases recorded, three fourth (74\%) were living in African countries (4). About 6.1 million cases were of extra pulmonary tuberculosis (EPTB), which represents $15 \%$ of the global TB burden. This ranges from $8 \%$ in the western pacific region to $23 \%$ in the eastern Mediterranean region (5). Genitourinary TB is also one of the most common forms and responsible for $15-40 \%$ of EPTB (6). Ethiopia has reported a higher than average proportion of EPTB. Urinary tract tuberculosis (UTTB) is also one of the most common types and a severe form of EPTB in clinical practice. About $20 \%$ of the EPTB cases reported annually are UTTB. Urinary tract tuberculosis results from the spread of MTB at the time of primary pulmonary infection or reactivation of old pulmonary infection. Reactivation is common in people suffering from immunodeficiency or those taking steroids. Then, The infection spreads hematogenously into different organ like kidney (7).

Early detection of bacilli in clinical samples and starting sufficient treatment is extremely important to reduce spread of the epidemic and mortality rate $(8,9)$. Due to delayed diagnosis, the morbidity and mortality rate is increased (10). Yet, the diagnosis of UTTB is difficult, because its symptoms are similar to other bacterial infections, and the bacterial load in non-respiratory specimens is generally very low (11). It is also the major cause for diagnosis delay and unfavorable treatment outcome of UTTB patients (12).

The Gene X-pert MTB/RIF is currently endorsed by WHO for pulmonary tuberculosis (PTB) diagnosis in children and in selected group for the EPTB diagnosis. The Gene X-pert MTB/RIF assay is a cartridgebased, semi-automated, rapid molecular assay, which permits rapid TB diagnosis through detection of the Deoxy Nucleic Acid (DNA) of MTB and simultaneous identification of a majority of the mutations that confer rifampicin resistance (rpoB gene mutation (13). The MTB-DR plus is a multiplex DNA amplification test for routine identification of TB and detection of genomic sequences of anti-tuberculosis drug resistance (4). As a result, significant improvements have been made in the diagnosis of TB since the WHO updated its recommendation to use the rapid test called the Gene X-pert MTB/RIF assay for not only PTB but also EPTB in adults and children (6).

Conventional methods like Zehienelson (ZN) and fluorescence microscopy (FM) method are commonly used to detected PTB and some types of EPTB. But these methods have lower sensitivity and specificity than the Gene X-pert MTB/RIF assay. Data on diagnosis UTTB by Gene X-pert MTB/RIF assay from developing countries particularly from Africa including Ethiopia is yet limited mainly due to resource constraints and unavailability of Gene X-pert MTB/RIF assay. Considering the limited diagnostic and treatment possibilities in resource-poor countries, reliable surveillance data on the diagnosis of EPTB 
especially UTTB in different African countries including Ethiopia is urgently required. Therefore, this study was primarily aimed to compare Gene X-pert MTB/Rifampicin assay and ZN. Also evaluates FM in the diagnosis of UTTB among TB positive and suspected urinary tract tuberculosis patients.

\section{Methods And Materials}

\section{Study Area}

This study was conducted at University of Gondar Specialized Referral Hospital (UoG-SRH), which is located in Gondar town. The town is found $737 \mathrm{~km}$ far from Addis Ababa, the capital city of Ethiopia, and $180 \mathrm{~km}$ far from Bahir-dar (capital city of Amhara region). The town is located at $12^{\circ} 361 \mathrm{~N} 37^{\circ} 281 \mathrm{~W}$ latitude and longitude with an elevation of 2133 meters above sea level. According to the central and statistical agency of Ethiopia report in 2015, the town has twelve sub-city, twenty-two urban and eleven rural Kebeles with a total population of 323,900. Gondar town has 8 health centers, 21 private clinics, and one Specialized Referral Hospital in the town. The hospital provides health care service for more than 7 million people living in central Gondar zones and the surrounding zones and for some parts of the neighboring region.

\section{Study design and period}

A hospital-based cross-sectional study was conducted to compare the detection rate of Gene X-pert MTB/RIF assay, and conventional methods (ZN and FM microscopy) in the diagnosis of UTTB among pulmonary TB positive, and who were presumptive of UTTB cases at University of Gondar Specialized Referral Hospital, from February 2020 to June 2020 G.C, Northwest Ethiopia.

\section{Populations}

\section{Source and study population}

The source population for this study was all patients who attended the UoG-SRH during study period while the study population was who were MTB positive patients, and presumptive UTTB patients from February 2020 to June 2020 G.C.

\section{Inclusion criteria and exclusion criteria}

All patients attending at UoG-SRH who had UTTB complaints and pulmonary TB positive were participated in the study during the study period. Whereas, patients who were diagnosed for UTTB, and who were critically ill and unable to give consent or a urine sample were excluded from the study.

\section{Sample size determination and sampling technique}

The sample size was determined using CLSI/NCCLS recommended sample size calculation for method comparison, which is $60-100$ (14). Accordingly, the minimum sample size was 60 and $5 \%$ reserve, the 
total sample size was 64 . To include study subjects in this study, non-randomized purposive sampling technique was used.

\section{Operational definitions}

Tuberculosis is an infectious disease caused by MTB complex (15). Gene X-pert MTB/RIF is an automated real-time PCR system that simultaneously detects TB and resistance to Rifampicin (9).

Urinary TB is one of the most common types of EPTB and is also considered as a severe form of EPTB in clinical practice. Patients fulfill the following criteria: Clinical suspected cases plus one or more of the following: positive smear microscopy examination or positive Gene X-pert MTB/RIF. Or clinical suspected cases plus positive pathological examination.

Suspected cases UTTB: having one of the following symptoms these are frequent voiding, dysuria, pyuria, pain (back, flank or abdominal), and microscopic or macroscopic hematuria (16).

\section{Data collection and laboratory methods}

\section{Socio-demographic data and specimen collection}

A pre-tested and structured questionnaire was used to obtain socio-demographic characteristics of study subjects. Appropriate urine specimens were collected by a laboratory technologist by instructing the clients to collect approximately $10 \mathrm{ml}$ to $15 \mathrm{ml}$ mid-stream urine in a clean, leak-proof sterile plastic cup. Then, mid-stream urine sample was collected from study participants.

\section{Specimen processing and laboratory procedures}

\section{X-pert MTB/RIF assay procedure}

A urine sample was immediately transported to the laboratory and centrifuge at $3000 \mathrm{rpm}$ for 5 minutes. From the centrifuged samples the supernatant was discarded. Then, the sediment was diluted with sample reagent in a 1:2 ratio to make the sample liquefy, kill the bacilli, and lyse the cell. Then, $2 \mathrm{ml}$ of the liquefied sample was dispensed to the cartridge which contains the components of the PCR (primers, nucleotides, DNA polymerase, buffer, and DNA Template) by using a sterile pasture pipette. Finally, the cartridge was inserted into the Gene X-pert MTB/RIF assay instrument. Then, the results were displayed within $2 \mathrm{hrs}$. Here, all the necessary materials (the cartridge, pasture pipette, and the sample reagent) is contained in a manufacturer kit.

\section{Ziehl-Nielsen (ZN) staining procedure}

Urine samples were collected in the microbiology laboratory and immediately centrifuged at $3000 \mathrm{rpm}$ for 5 minutes. From the centrifuged samples the supernatant was discarded and the sediment was smeared. Then, the smeared slides were arranged and flooded with filtered $0.1 \%$ carbolfuchsin. After the smears heated with a flame and formed the steam, they were allowed to stain for 5 minutes, washed with water. Then, they were decolorized with $3 \%$ acid alcohol for 3 minutes, rinsed with water, and drained. The 
decolorized slide was counterstained with $0.1 \%$ methylene blue solution for 1 minute and rinsed with water. The smear was allowed to air dry and examined microscopically using the oil immersion (100x) objective (17).

\section{Fluorescence microscopy staining procedure}

The collected urine samples immediately centrifuged at $3000 \mathrm{rpm}$ for 5 minutes. From the centrifuged samples the supernatant was discarded and the sediment was smeared. The smears were flooded with filtered $0.1 \%$ auramine for at 20 minutes, rinsed with water and drained. Then the stained slides were decolorized by acid alcohol decolorizing solution (0.5\%) for 30 to 60 seconds, rinsed with water, and drained. After that, the slides were counter-stained with $0.5 \%$ potassium permanganate counterstain for a maximum of 1 minute and rinsed with water. The smears were allowed to air dry and examined microscopically using the dry (40x) objective lens of a light emitting diodes illumination-based fluorescence microscope (Zeiss primo star ilED) (17).

\section{Data Quality Control}

The quality of the questioner was pre-tested before the actual study begins in the poly health center to make sure that whether the questionnaire was appropriate and understandable by patients. The collected data were checked daily for consistency and accuracy. Investigators also followed data collection process. Microscope examination and Gene X-pert was prepared according to the manufacturer's instruction following standard operational procedure (SOPs). The reliability of the study findings was guaranteed by implementing quality control measures throughout the whole process of the laboratory work (by using homemade and commercial known positive and negative samples). All materials, equipment, and procedures were adequately controlled based on pre-analytical, analytical and postanalytical stages of quality assurance that are incorporated in SOPs of the UoG-SRH TB Laboratory. The reliability of microscopic examination and Gene X-pert checked by positive and negative control samples.

\section{Data analysis and interpretation}

Data were entered using EPI-Info version-3.5.3 then transported to SPSS version 23 for analysis. Then, descriptive statistics were summarized as percentages, means, and standard deviations and presented with figures and tables. Each of the outcome variables was computed with each independent variable.

\section{Results}

\section{Socio-demographic characteristics}

A total of 64 study participants were enrolled in this study. Of them, $64.2 \%(41 / 64)$ were males and the $29.69 \%$ of the study participants were in the age group of 31-45 years. From the study participants, $71.9 \%$ (46/64) were rural residence and $51.6 \%$ (33/64) were farmers in their occupation, and $57.8 \%$ $(37 / 64)$ were illiterate (Table 1$)$. 
Table 1

Socio demographic characteristics of study participants at UoG-SRH Northwest Ethiopia in 2021.

\begin{tabular}{|c|c|c|c|}
\hline \\
\hline Variable & & Frequency & Percent \\
\hline \multirow[t]{2}{*}{ Sex } & Male & 41 & 64.1 \\
\hline & Female & 23 & 35.9 \\
\hline \multirow[t]{2}{*}{ Residence } & Rural & 46 & 71.9 \\
\hline & Urban & 18 & 28.1 \\
\hline \multirow[t]{5}{*}{ Age in year } & $>16$ & 14 & 21.9 \\
\hline & $16-30$ & 18 & 28.1 \\
\hline & $31-45$ & 28 & 43.8 \\
\hline & $46-60$ & 4 & 6.3 \\
\hline & $>60$ & 18 & 28.1 \\
\hline \multirow[t]{2}{*}{ Religion } & Orthodox & 57 & 89.1 \\
\hline & Muslim & 7 & 10.9 \\
\hline \multirow[t]{5}{*}{ Occupation } & Farmer & 33 & 51.6 \\
\hline & Civil servant & 13 & 20.3 \\
\hline & Private-worker & 8 & 12.5 \\
\hline & House wife & 7 & 10.9 \\
\hline & Student & 3 & 4.7 \\
\hline \multirow[t]{4}{*}{ Educational status } & Illiterate & 37 & 57.8 \\
\hline & Secondary school & 17 & 26.6 \\
\hline & preparatory school & 3 & 4.7 \\
\hline & University or college & 7 & 10.9 \\
\hline \multirow[t]{3}{*}{ Marital status } & Single & 19 & 29.7 \\
\hline & Married & 43 & 67.2 \\
\hline & Divorced & 2 & 3.1 \\
\hline
\end{tabular}

\section{Gene X-Pert and conventional examination method}

Among 64 study participants, 4.69\% (3/64) were positive for urinary tuberculosis by G-expert and also $1.56 \%$ (1/64) was positive by FM. But from all study participants there was no positive finding by ZN microscopy (Table 2). 
Table 2

Gene x-pert and conventional method results among study participants at UoG-SRH Northwest Ethiopia in 2021.

\begin{tabular}{|llll|}
\hline Variable & & Frequency & Percent \\
\hline Diagnosis & TB & 51 & 79.7 \\
\cline { 2 - 4 } & Drug resistance TB & 13 & 20.3 \\
ZN microscopic examination result & Negative & 64 & 100 \\
\cline { 2 - 4 } FM microscopy examination result & Positive & 0 & 0 \\
\cline { 2 - 4 } & Negative & 63 & 98.4 \\
\cline { 2 - 4 } Gene X-pert result MTB & Positive & 1 & 1.6 \\
& Detected & 3 & 4.7 \\
\cline { 2 - 4 } & Not detected & 61 & 95.3 \\
\cline { 2 - 4 } & & & \\
\hline
\end{tabular}

From all study participants $4.69 \%$ (3/64) were positive for UTTB. Out of positive cases by Gene X-pert assay, $66.7 \%(2 / 3)$ of the positive cases was from age $16-30$ years and $33.3 \%(1 / 3)$ was with $46-60$ age range. Among the study participants residence $66.7 \%$ (2/3 rural and $33.3 \%(1 / 3)$ urban were positive for Gene x-pert. Among treatment history of the patients $66.7 \%$ (2/3) were new and 33.3\% (1/3) were first-line treatment (Table 3). 
Table 3

Crosstab result of Gene X-pert at University of Gondar Specialized Referral Hospital Northwest Ethiopia in 2021.

\begin{tabular}{|c|c|c|c|c|}
\hline \multirow[t]{2}{*}{ Variables } & & \multicolumn{3}{|c|}{ Characteristics } \\
\hline & & Detected & $\begin{array}{l}\text { Not } \\
\text { detected }\end{array}$ & Total \\
\hline \multirow[t]{2}{*}{ Residence } & Rural & $2(3.13 \%)$ & $44(68.75 \%)$ & $46(71.89)$ \\
\hline & Urban & $1(1.56)$ & $17(26.56 \%)$ & $18(28.13 \%)$ \\
\hline \multirow[t]{4}{*}{ Educational status } & Illiterate & $2(3.13 \%)$ & $35(54.69 \%)$ & $37(57.81 \%)$ \\
\hline & Secondary school & $0(0 \%)$ & $17(26.56 \%)$ & $17(26.56 \%)$ \\
\hline & Preparatory school & $0(0 \%)$ & $3(4.69 \%)$ & $3(4.69 \%)$ \\
\hline & University or college & $1(1.56 \%)$ & $6(9.34 \%)$ & $7(10.94 \%)$ \\
\hline \multirow[t]{3}{*}{ Marital status } & Married & $2(3.13 \%)$ & $41(64.06 \%)$ & $43(67.19 \%)$ \\
\hline & Single & $1(1.56 \%)$ & $18(28.13 \%)$ & $19(29.69 \%)$ \\
\hline & Divorced & $0(0 \%)$ & $2(3.13 \%)$ & $2(3.13 \%)$ \\
\hline \multirow[t]{2}{*}{ TB treatment history } & New & $2(3.13 \%)$ & $51(79.69 \%)$ & $53(82.81 \%)$ \\
\hline & First line & $1(1.56 \%)$ & $10(15.63 \%)$ & $11(17.19 \%)$ \\
\hline \multirow{3}{*}{$\begin{array}{l}\text { TB patient registration } \\
\text { group }\end{array}$} & New & $2(3.13 \%)$ & $53(82.81 \%)$ & $55(85.94 \%)$ \\
\hline & Relapse & $0(0 \%)$ & $7(10.94 \%)$ & $7(10.94 \%)$ \\
\hline & $\begin{array}{l}\text { Treatment after lose to follow } \\
\text { up }\end{array}$ & $1(1.56 \%)$ & $1(1.56 \%)$ & $2(3.13 \%)$ \\
\hline
\end{tabular}

\section{Sensitivity and specificity of FM and ZN microscopy result compared to gene X-pert}

While comparing the FM with Gene X-pert, the sensitivity and specificity was $33.3 \%$ and $100 \%$. Also when we compare the ZM with Gene X-pert the ZN microscopy the sensitivity and specificity were $0 \%$ and $100 \%$ (Tables 4 and 5). 
Table 4

Comparison of Gene X-pert with ZN microscopy of the study participants at UoG-SRH Northwest

Ethiopia in 2021.

\begin{tabular}{|llll|}
\hline ZN Microscope & \multicolumn{2}{l}{ Gene X-Pert } & Total \\
\cline { 2 - 3 } & Positive & Negative & \\
\hline Positive & 0 & 0 & 0 \\
\hline Negative & 3 & 61 & 64 \\
\hline Total & 3 & 61 & 64 \\
\hline
\end{tabular}

Table 5

Comparison of Gene X-pert with FM microscopy of the study participants at UoG-SRH Northwest Ethiopia in 2021.

\begin{tabular}{|lccc|}
\hline FM Microscopy & \multicolumn{2}{l}{ Gene X-Pert } & Total \\
\cline { 2 - 3 } & Positive & Negative & \\
\hline Positive & 1 & 0 & 1 \\
\hline Negative & 2 & 61 & 63 \\
\hline Total & 3 & 61 & 64 \\
\hline
\end{tabular}

\section{Discussion}

In this study, the overall detection rate of urinary TB using Gene X-pert, FM, ZN among study participants were $4.69 \%$ (3/64), 1.56\% (1/64), 0\% (0/84), respectively. This result showed that out of 64 PTB infected individuals by using Gene X-pert three individuals developed UTTB. The TB bacilli usually affects adults between the second and fourth decades of life. In the current study, all three cases were among the adult age group. These UTTB infected individuals can complicate a kidney disease. Because the kidney is the most common site that is attacked by UTTB. Occasionally, the patient who had UTTB might be advanced disease to obstructive uropathy, bladder defects, and loss of kidney function. A study conducted among UTTB cases showed that a history of PTB was a risk to develop UTTB. This is because patients who were infected with PTB and who were immune-compromised can be susptible to spread the bacteria via the blood and it can affect different organs like the kidney and lead to developed UTTB (18).

The current study finding was lower than to a study conducted in Iran which, was 9.4\% (79/838) of extra pulmonary specimens (8). Also, the current study detection rate of Gene X-pert MTB/RIF was lower than a study conducted in China, Pakistan, Morocco with the detection rate of Gene X-pert MTB/RIF was 40.5\%, $77.4 \%$, and 20.59 , respectively (12) (19). The sensitivity and specificity of study conducted in Morocco were 79,3 and $90.3 \%$, respectively (4). Also, high detection rate was found in Jharkhand showed 96.43 (20). Similarly, detection rates for microscopy, Gene X-pert MTB/RIF was 12.88 , and 20.59 , respectively 
(21). This significant difference might be due to that the sample size, the capacity of clinical provider case identification at the clinical level. Statistical analysis revealed that the sensitivity of Gene X-pert was significantly higher than that of AFB microscopy $(P<0.001)(12)$.

In the current study, the FM UTTB detection rate compared to Gene X-pert MTB/RIF was $33.3 \%(1 / 3)$. Our finding was higher than a study conducted in Morocco which was $12.88 \%$ positivity rates for FM. In contrast which was lower than the study in India that was $46 \%$ (5). The discrepancy may be due to sample size, population diversity, and geographical location. The current study and study conducted before indicated that FM microscopy has lower detection rate than Gene X-pert MTB/RIF even if the results have some variety.

In this study, the sensitivity and specificity of urinary ZN microscopy compared to Gene X-pert among suspected TB cases were $0 \%$ and $100 \%$, respectively. This showed that the detection rate of ZN microscopy was very lower than Gene X-pert MTB/RIF, and FM microscopy. But there were no false positive results. The current study result was lower than the cross-sectional study conducted in Dhaka $61 \%(22)$. This variation might be due to that reagent condition likely environmental factor and storage area.

Among study participants, adult males were highly affected than females. This is a similar study with a study conducted in Indian (5). This might be due to the adult males are more exposed to the environmental factors, high workload, and wide range of mobility than the female and younger age groups. The strength of this study is assess UTTB by following SOP to all the three method (FM, ZN, and Gene X-pert). The major limitation of this study was due to resource constraint we did not perform TB culture which may improve positive rate and it was also possible to compare the method with Gene X-pert and conventional methods.

\section{Conclusion And Recommendation}

The overall detection rate of urinary tract MTB by Gene X-pert, fluorescence microscopy, and ZN microscopy was $4.69 \%, 1.56 \%$, and $0 \%$. The prevalence of UTTB was higher in urban residents and male study participants. On the other hand, it is also higher in illiterate and married participants. Also, the sensitive to the conventional method like FM, and ZN compared to Gene X-pert MTB/RIF was 33.3\% and $0 \%$. Gene $X$-pert had better performance than conventional method in detecting UTTB from urine samples and could be considered for the diagnosis of UTB. As a result, the urine sample can be one of the diagnosis samples for urinary tract tuberculosis.

Based on the study finding the following recommendations are needed to health professionals, any stack holders, and researchers. The ministry of health/regional health bureau should set guidelines on how to use G-expert for UTTB in the urine sample and availed G-expert should be distributed to all health facilities. For the Regional Health Bureau, update health care workers about urine for G-expert of UTTB diagnosis could develop. For the health worker, it is better to use Gene X-pert for UTB detection rather than the conventional methods. 


\section{List Of Abbreviations}

DNA- Deoxyribonucleic acid, EPTB: External Pulmonary Tuberculosis, FM: Florescent Microscopy,MTB: Mycobacterium tuberculosis, PTB: Pulmonary Tuberculosis, Rif: Rifampicin, SOP: Standard Operational Definition, SPSS-Statistical Package for Social Sciences, TB: Tuberculosis, UoGUniversity of Gondar, UoG-SRH: University of Gondar Specialized Referral Hospital, UTB-Urinary Tuberculosis, WHO-World Health Organization, ZN-Ziehl Nelson.

\section{Declarations}

\section{Ethical consideration}

The study was conducted after obtaining ethical clearance from the ethical review committee of the institute of biotechnology, University of Gondar. A legal Permission letter was obtained from the UoG-SRH before data collection. The participants recruited to the study were informed about the objectives of the study and their participation was voluntary and participants have the right to withdraw at any point from the study. Written informed consent was obtained from all study participants and assent was also obtained from children's parents or guardians involved in the study. All the laboratory examinations of urine samples were done as free from charge and confirmed positives were given appropriate treatment. Information obtained at any course of the study was kept confidential. Confidentiality was maintained by numeric coding of specimens and questionnaires. Further positive finding for UTTB was reported to patients and their medical doctor to take an appropriate treatment and follow-up.

\section{Competing interest}

The authors declare that there is no conflict of interests regarding the publication of this manuscript.

\section{Authors' contributions}

MA and AT participated in this study by data feeding, performed the statistical analysis, and drafted the manuscript. All the authors (MW, DE, and NB) collect the data, interpreted the data, and read and edits the manuscript. All authors read and approved the final manuscript.

\section{ACKNOWLEDGMENT}

We would like to thank to both referral hospital for their providing the necessary information during the data collection.

\section{Funding}

The authors received no specific funding for this work

\section{Availability of data and materials}


All data supporting these findings is contained within the manuscript.

\section{Consent for publication}

Not applicable. This manuscript does not contain any individual persons' data

\section{References}

1. Shi J, Dong W, Ma Y, Liang Q, Shang Y, Wang F, et al. GeneXpert MTB/RIF outperforms mycobacterial culture in detecting mycobacterium tuberculosis from salivary sputum. BioMed research international. 2018;2018.

2. Altiparmak MR, Trabulus S, Balkan II, Yalin SF, Denizli N, Aslan G, et al. Urinary tuberculosis: a cohort of 79 adult cases. Renal failure. 2015;37(7):1157-63.

3. Hamada Y, Glaziou P, Sismanidis C, Getahun H. Prevention of tuberculosis in household members: estimates of children eligible for treatment. Bulletin of the World Health Organization. 2019;97(8):534.

4. Mechal Y, Benaissa E, Benlahlou Y, Bssaibis F, Zegmout A, Chadli M, et al. Evaluation of GeneXpert MTB/RIF system performances in the diagnosis of extrapulmonary tuberculosis. BMC infectious diseases. 2019;19(1):1-8.

5. Koul AN, Kassana BA, Rather AR. Utility of GeneXpert in the diagnosis, reliance on urine microscopy and clinical characteristics of genitourinary tuberculosis at a tertiary care hospital. Indian journal of medical microbiology. 2018;36(1):93-6.

6. Chen Y, Wu P, Fu L, Liu Y-h, Zhang Y, Zhao Y. Multicentre evaluation of Xpert MTB/RIF assay in detecting urinary tract tuberculosis with urine samples. Scientific reports. 2019;9(1):1-6.

7. Denkinger CM, Schumacher SG, Boehme CC, Dendukuri N, Pai M, Steingart KR. Xpert MTB/RIF assay for the diagnosis of extrapulmonary tuberculosis: a systematic review and meta-analysis. European Respiratory Journal. 2014;44(2):435-46.

8. Allahyartorkaman M, Mirsaeidi M, Hamzehloo G, Amini S, Zakiloo M, Nasiri MJ. Low diagnostic accuracy of Xpert MTB/RIF assay for extrapulmonary tuberculosis: A multicenter surveillance. Scientific reports. 2019;9(1):1-6.

9. Theron G, Peter J, Calligaro G, Meldau R, Hanrahan C, Khalfey H, et al. Determinants of PCR performance (Xpert MTB/RIF), including bacterial load and inhibition, for TB diagnosis using specimens from different body compartments. Scientific reports. 2014;4(1):1-10.

10. Mavenyengwa RT, Shaduka E, Maposa I. Evaluation of the Xpert® MTB/RIF assay and microscopy for the diagnosis of Mycobacterium tuberculosis in Namibia. Infectious diseases of poverty. 2017;6(1):1-5.

11. Tortoli E, Russo C, Piersimoni C, Mazzola E, Dal Monte P, Pascarella M, et al. Clinical validation of Xpert MTB/RIF for the diagnosis of extrapulmonary tuberculosis. European Respiratory Journal. 2012;40(2):442-7. 
12. Pang Y, Shang Y, Lu J, Liang Q, Dong L, Li Y, et al. GeneXpert MTB/RIF assay in the diagnosis of urinary tuberculosis from urine specimens. Scientific reports. 2017;7(1):1-6.

13. Maynard-Smith L LN, Peters JA, Lawn SD. Diagnostic accuracy of the Xpert MTB/RIF assay for extrapulmonary and pulmonary tuberculosis when testing non-respiratory samples: a systematic review. BMC infectious diseases. 2014 Dec;14(1):1-5.

14. NCCLS. Method Comparison and Bias Estimation Using Patient Samples: Approved Guideline Second edition. NCCLS document EP9-A2 (ISBN 1-56238-472-4). NCCLS WVR.

15. Singh UB, Pandey P, Mehta G, Bhatnagar AK, Mohan A, Goyal V, et al. Genotypic, phenotypic and clinical validation of GeneXpert in extra-pulmonary and pulmonary tuberculosis in India. PLoS One. 2016;11(2):e0149258.

16. Gounder CR, Tendesayi K, Wada NI, Mngomezulu V, Charalambous S, Hanifa Y, et al. Diagnostic accuracy of a urine lipoarabinomannan enzyme-linked immunosorbent assay for screening ambulatory HIV-infected persons for TB. Journal of acquired immune deficiency syndromes (1999). 2011;58(2):219.

17. Dzodanu EG AJ, Acheampong DO, Dadzie I. Diagnostic yield of fluorescence and Ziehl-Neelsen staining techniques in the diagnosis of pulmonary tuberculosis: a comparative study in a District Health Facility. Tuberculosis research and treatment. 2019 Apr 10;2019.

18. Amaya-Tapia G, Aguirre-Avalos G. Urinary Tract Tuberculosis. Tuberculosis. 2018 Sep $26 .$.

19. Munir MK, Rehman S, Aasim M, Iqbal R, Saeed S. Comparison of Ziehl Neelsen microscopy with GeneXpert for detection of Mycobacterium tuberculosis. IOSR J Dent Med Sci. 2015;14(11):56-60.

20. Kumar M, Bharati OP, Sharma AK, Prasad A, Seema K. Comparative Study Of Gene XPERT MTB/RIF, ZIEHL NEELSEN Smear Microscopy And MGIT 960 Culture In Samples Of Suspected Pulmonary Tuberculosis In A Tertiary Care Hospital Of Jharkhand.

21. Zeka AN, Tasbakan S, Cavusoglu C. Evaluation of the GeneXpert MTB/RIF assay for rapid diagnosis of tuberculosis and detection of rifampin resistance in pulmonary and extrapulmonary specimens. Journal of clinical microbiology. 2011;49(12):4138-41.

22. Hossain MM, Amin MR, Kabir A, Ahmed MZ, Gomes LC, Ahmed S, et al. Front-loaded (FL) microscopic approach against Conventional approach for detecting Acid Fast Bacilli (AFB) in sputum. Journal of Medicine. 2020;21(1):36-40. 